\title{
Realizable Minimally Invasive 1-Day Lumbar Interbody Fusion Surgery: No General Anesthesia, No Hemovac Insertion, No Skin Suture Surgery, and Early Ambulation
}

\author{
Jun-Ho Lee ${ }^{1}$, Hyeun-Sung Kim², Jee-Soo Jang ${ }^{2}$, Il-Tae Jang ${ }^{3}$, Seong Hoon $\mathrm{Oh}^{1}$, Jin-Uk Kim ${ }^{1}$ \\ ${ }^{1}$ Department of Neurosurgery, Nanoori Incheon Hospital, Incheon, \\ ${ }^{2}$ Department of Neurosurgery, Nanoori Suwon Hospital, Suwon, \\ ${ }^{3}$ Department of Neurosurgery, Nanoori Hospital, Seoul, Korea
}

Corresponding Author: Hyeun-Sung Kim, $\mathrm{MD}, \mathrm{PhD}$ Department of Neurosurgery, Nanoori Suwon Hospital, 295, Jungbudaero, Yeongtong-gu, Suwon-si 16503, Korea

Tel: $+82-2-1688-9797$

Fax: +82-31-8065-9797

E-mail: neuros@hanmail.net

Received: April 26, 2016 Revised: May 15, 2016 Accepted: June 1, 2016

\begin{abstract}
Objective: The lumbar interbody fusion surgery, patients commonly have severe pain, requiring adequate bed rest for a long time. We performed a 1-day minimally invasive spine (MIS) lumbar interbody fusion that required no hemovac insertion and no skin suture and led to early ambulation. Here, we report the surgical procedure and results. Methods: This study was designed as a retrospective review. From January 2013 to August 2014, 49 patients who received the MIS TLIF for 1-day MIS lumbar interbody fusion surgery were included in this study. The surgical procedures performed were as follows: (1) epidural catheter insertion; (2) midline subdermal dissection procedure; (3) MIS TLIF; (4) bleeding control procedure; (5) percutaneous transpedicular screwing; (6) tight subdermal plan suture; (7) skin sealing procedures. Postoperatively, wound dressing was not needed. Epidural catheter was removed on the second day after the operation. Results: Average intraoperative bleeding was $128.6 \mathrm{~mL}$ per level. The average operation time was $78.9 \mathrm{~min}$. per level. An average midline skin incision was $2.8 \mathrm{~cm}$ per level. The possible ambulation time was 0.94 \pm 0.88 day. The discharge time after antibiotic injection for 3 days was $4.88 \pm 1.51$ days. In the corresponding order of preoperative and immediate postoperative, 3-month, 6-month, and final followup, Postoperative VAS (back), VAS (leg) and ODI improved significantly immediate postoperatively $(p<0.0001)$. Postoperatively, there was no cases of revision due to hematoma. Conclusion: The results indicated good clinical results of the 1-day minimally invasive lumbar interbody fusion surgery, without any serious complications.
\end{abstract}

Key Words: Minimally invasive, Transforaminal, Lumbar interbody fusion, 1-day surgery

\section{INTRODUCTION}

The incidence of degenerative spinal diseases that require lumbar interbody fusion surgery has increased with an increase in the elderly population. However, following lumbar interbody fusion surgery, patients commonly have severe pain and require long periods of adequate bed rest. Moreover, associated complications can occur, leading to a delay in rehabilitation.

An extensive body of research supports Minimally Invasive Surgical (MIS) techniques as effective in decreasing postsurgical morbidity and improving postoperative recovery ${ }^{13)}$.

However, because of the risk of complications, patients who are in need of fusion surgery, especially the elderly, tend to avoid surgery. Furthermore, shortening of the length of surgery and hospitalization is an important factor in the onset and recovery of complications in patients with a systemic disease.

In fact, a prior study has reported outcomes following 1-day fusion surgery ${ }^{15)}$. However, in this study, although patients were discharged on the same day as surgery, it did not mean that care was also terminated. Rather, this study compared and verified that same-day discharge did not cause complications. In fact, stitch removal and patient care were managed in outpatient appointments after discharge. Thus, the patients were not truly discharged but were transferred to home care. The fact that the patients were still in need of stitch removal and hospital 
care signified that the patients were not completely discharged from the hospital.

Ambulation is a critical aspect of rehabilitation and positively affects patients' recovery. In fact, the main reasons patients stay in hospital are as follows: (1) postoperative pain management, (2) management and removal of any drainage tubes, and (3) postoperative wound care. Thus, if these procedures were unnecessary, true 1-day fusion surgery would be possible.

We performed a 1-day MIS lumbar interbody fusion that did not require Hemovac insertion or postoperative sutures and allowed early ambulation. Here, we report the surgical procedure and results.

\section{MATERIALS AND METHODS}

This study was designed as a retrospective review of clinical and surgical parameters. From January 2013 to August 2014, 49 patients who underwent 1-day MIS transforaminal lumbar interbody fusion (TLIF) surgery were included in this study.

All patients underwent MIS TLIF using an MIS retractor system (Tubular/Caspar/Taylor) and MIS decompression technique (unilateral decompression/bilateral decompression/unilateral approach bilateral decompression). Two cases were being treated for foraminal stenosis, one for recurrent Herniated Nucleus Pulposus (HNP), 13 for spinal stenosis, and 33 for spondylolisthesis.

\section{Patients}

This study examined surgeries conducted between January 2103 and August 2014 by reviewing the medical charts of 49 patients. Patients with spinal stenosis, spondylolisthesis, foraminal stenosis, or recurrent HNP were included in this study, all of which were in need of decompression, as well as pedicle screw fixation and fusion.

\section{Operative techniques}

Decompression was performed using the basic MIS TLIF procedure as described below.

\section{Technique for MIS TLIF}

The MIS TLIF procedure was performed on the symptomatic side. C-arm guidance was used to determine the disc space and to draw the lateral pedicle line in the fluoroscopic anterior posterior view. After a vertical skin incision in line with the lateral pedicle. After a complete facetectomy, the ligamentum flavum was removed to expose the lateral border of the ipsilateral nerve root. The retractor was angled medially, The patient was tilted laterally to decompress the contralateral side. Extensive decompression was performed, which included decompression of the central stenosis and contralateral side ${ }^{1,6,9)}$. A discectomy was also performed. A single, banana-shaped polyetheretherketone interbody cage filled with only autologous local bone was inserted. After interbody fusion, the retractor was removed, and the same procedure was repeated for each segment. Ipsilateral percutaneous pedicle screws were inserted through the same skin incision. Contralateral percutaneous pedicle screws were placed using a mirror incision under fluoroscopic guidance.

Additional surgical procedures performed were as follows:

1) Epidural catheter insertion for anesthesia and postoperative pain control; this allowed the procedure to be performed without a general anesthesia and controls postoperative pain effectively such that patients can ambulate shortly after surgery.

2) midline subdermal dissection procedure: this procedure can reduce the size of the skin incision, and the tension of the skin can reduce the risk of postoperative hematoma. Although it is a midline incision, dissection is performed at the subdermal level, and TLIF, decompression are performed via the paraspinal plane. PLIF, which comes in contact with the midline structure, can induce central accumulation of blood and eventually cause a postoperative hematoma (Fig. 1)

In addition, advances in MIS techniques for TLIF have reduced the incidence of complications and morbidity associated with conventional $\mathrm{TLIF}^{3)}$.

3) MIS TLIF procedure (unilateral/bilateral) (Fig. 2).

4) Percutaneous transpedicular screw insertion under the subdermal dissection plane (Fig. 3).

5) Blood loss control procedure: limiting blood loss is the most crucial step, as hemostasis is a key aspect in 1-day fusion surgery. Our procedures included the following: (A) meticulous bleeding control; (B) fibrinogen/thrombin-based collagen fleece bleeding control; (C) fluid-type anti-adhesive agent, which can stop venous bleeding using hydrostatic pressure; and (D) Gelfoam ${ }^{\mathbb{R}}$ covering, which acts as a barrier that stops bleeding that occurred outside the spinal canal, i.e., from the muscle, from coming into the canal (Fig. 4).

6) Tight subdermal plane suture (conjoined suture of split fascia and subdermal skin) (Fig. 5).

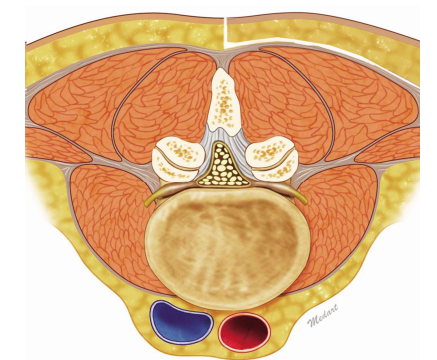

Fig. 1. Midline subdermal dissection.

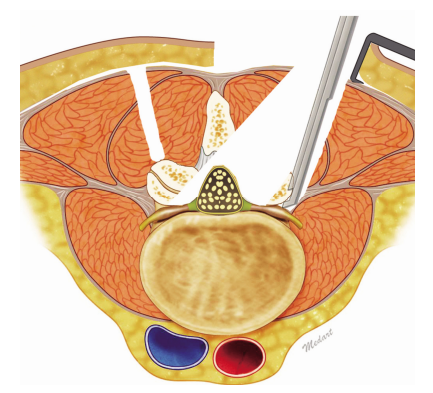

Fig. 2. Central decompression. 
7) Skin sealing procedures: secure skin and zip surgical skin closure systems (Fig. 6).

A postoperative wound dressing was not needed. The wounds were checked every 3-4 days. The epidural catheter was removed on the second day after the operation. Intravenous antibiotics were administered for 3 days after the operation.

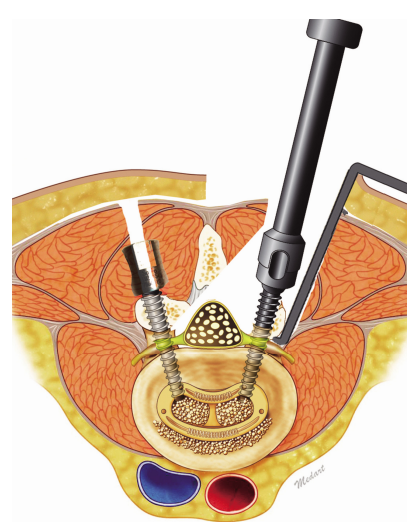

Fig. 3. Percutaneous transpedicular screw insertion.

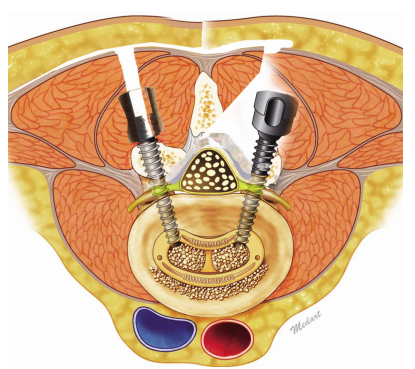

Fig. 4. Blood loss control procedure (fibrinogen fleece, fluid type antiadhesive agent, Gelform barrier)

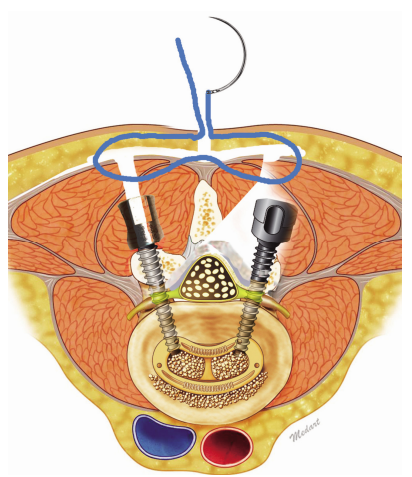

Fig. 5. Conjoined suture of fascia and subdermal skin.

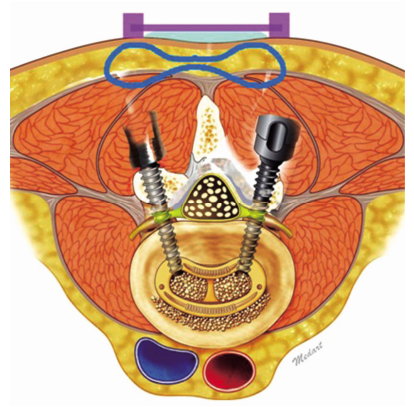

Fig. 6. Skin and zip surgical skin closure systems.
Epidural catheter insertion enables additional pain control. Even if an IV PCA is used, patients experience the most severe pain during the first two days post-operatively, which can be controlled continuously via the epidural catheter.

\section{RESULTS}

We examined surgery-related results, the intraoperative and postoperative conditions, postoperative complications, and clinical results by using the Visual Analogue Scale (VAS) and Oswestry Disability Index (ODI) immediately (1-2 days), and 1 month, 3 months, 6 months, and 12 months postoperatively.

\section{Demographics}

The mean age was $65.27 \pm 9.57$ years, and the sex ratio was 20:29 (male:female). The average follow-up period was $26.04 \pm 7.25$ months.

Regarding the number of segments involved in the operation, 33 patients underwent one segment; 13 patients, 2 segment; and 3 three patients, 3 segment operation (average: 1.39 \pm 0.61 segment).

\section{Clinical outcomes}

Average intraoperative bleeding was $178.47 \pm 73.70 \mathrm{~mL}$ (per level: $128.60 \mathrm{~mL}$ ). The average operation time was $109.49 \pm 32.71$ min (per level: $78.90 \mathrm{~min}$ ). Average midline skin incision length was $3.90 \pm 1.18 \mathrm{~cm}$ (per level: $2.80 \mathrm{~cm}$ ).

The possible ambulation time was $0.94 \pm 0.88$ day. The discharge time after 3 days' antibiotic administration was $4.88 \pm 1.51$ days.

The VAS (back) were as follows: $6.33 \pm 0.94,3.14 \pm 1.12,2.47 \pm$ $0.58,2.29 \pm 0.65$, and $2.31 \pm 0.77$; VAS (leg): $7.37 \pm 0.70,2.69 \pm 0.85$, $2.29 \pm 0.46,2.14 \pm 0.58$, and $2.24 \pm 0.80$; and ODI: $39.37 \pm 3.05,29.29 \pm$ $5.78,22.59 \pm 2.99,20.27 \pm 2.59$, and $18.63 \pm 3.13$ for the preoperative, immediate postoperative, and 3-month, 6-month, and 12-month follow-up values, respectively. Postoperative VAS (back), VAS (leg), and ODI improved significantly immediately postsurgery $(p<0.0001)$ (Table 1).

In terms of postoperative complications, there were two cases of transient motor weakness (both cases recovered sufficiently after the follow-up period), four requiring wound suture due to avulsion of the surgical field (all cases healed completely after the follow-up period), one of dural tear, and two of cage subsidence or implant failure. No cases required revision due to hematoma.

Table 1. Patient clinical outcomes

\begin{tabular}{|c|c|c|c|c|c|}
\hline & Preop & 1 day & 3 months & 6 months & Final $\mathrm{F} / \mathrm{U}$ \\
\hline & 6.33 & 3.14 & 2.47 & 2.29 & 2.31 \\
\hline VAS (Leg) & 7.37 & 2.69 & 2.29 & 2.14 & 2.24 \\
\hline ODI & 39.37 & 29.29 & 22.59 & 20.27 & 18.63 \\
\hline
\end{tabular}




\section{CASE}

56-year-old male who visited the hospital for severe, radiating low back pain and neurogenic claudication present for more than a few months. The 1-day postoperative MRI showed sufficient decompression with MIS TLIF. The patient was capable of walking by the afternoon on the day of surgery. He was capable of discharge after 1 postoperative day (Fig. 7-12).

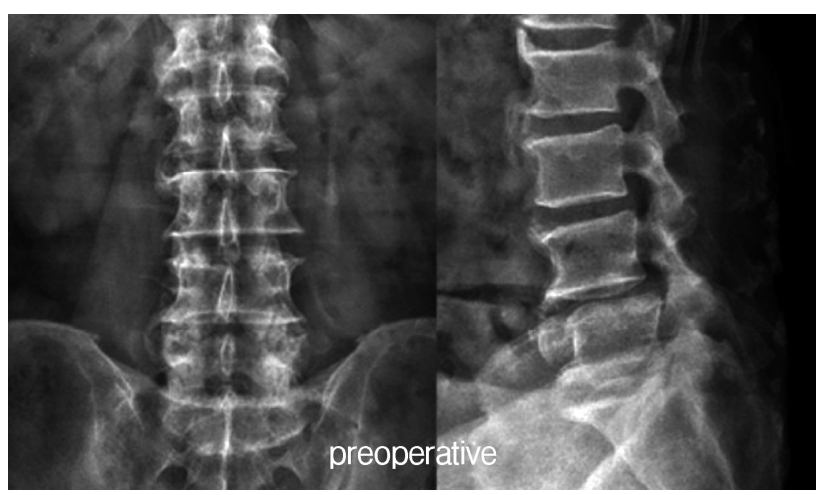

Fig. 7. Preoperative X-ray.

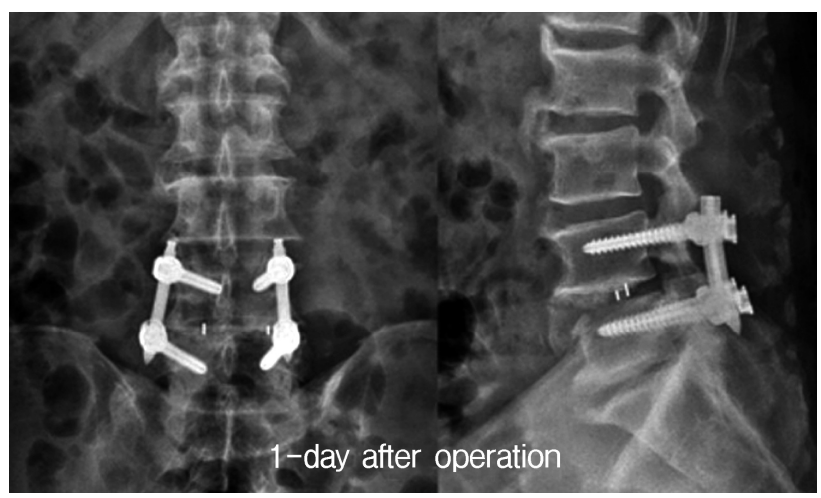

Fig. 8. 1-day postoperative $\mathrm{X}$-ray.

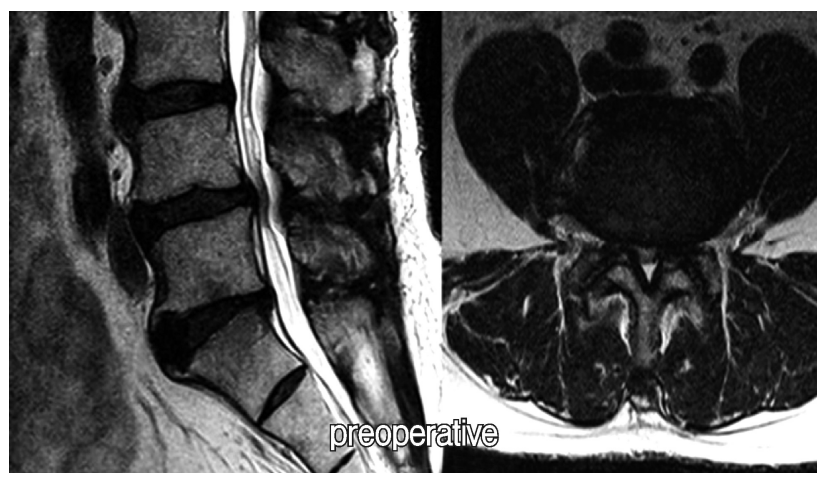

Fig. 9. Preoperative MRI.

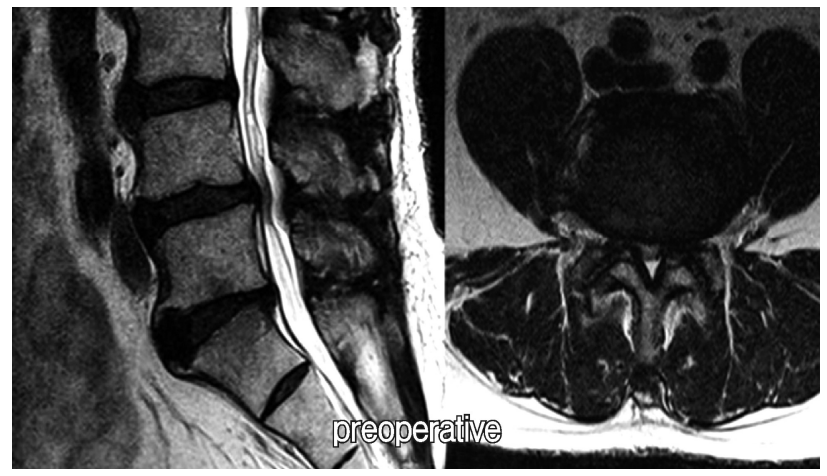

Fig. 10. 1-day postoperative MRI.

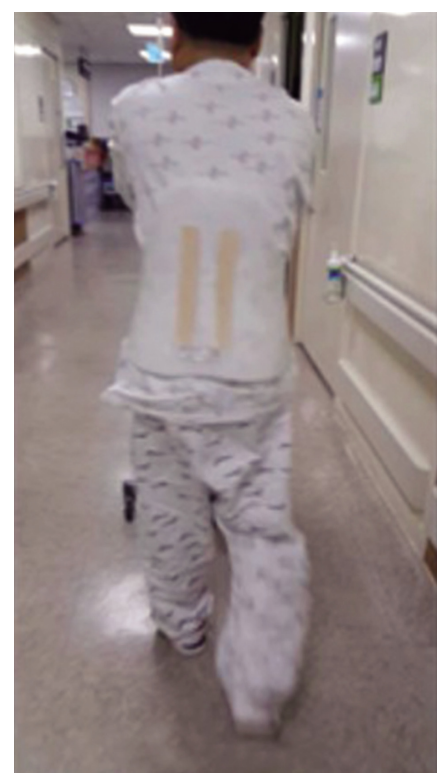

Fig. 11. Operation day ambulation.

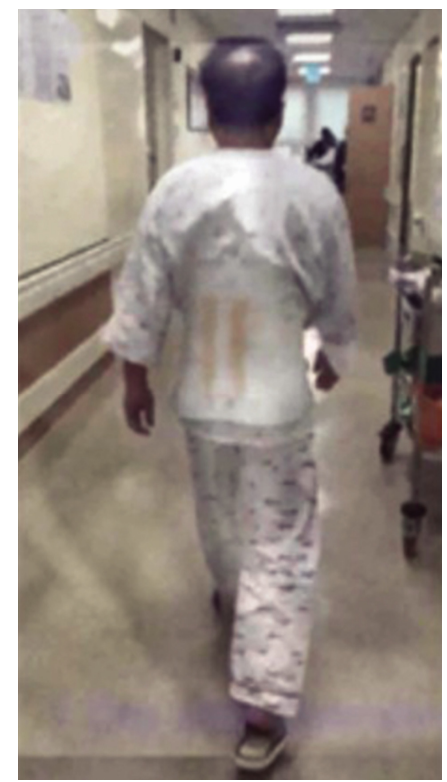

Fig. 12. 1-day postoperative day ambulation. 


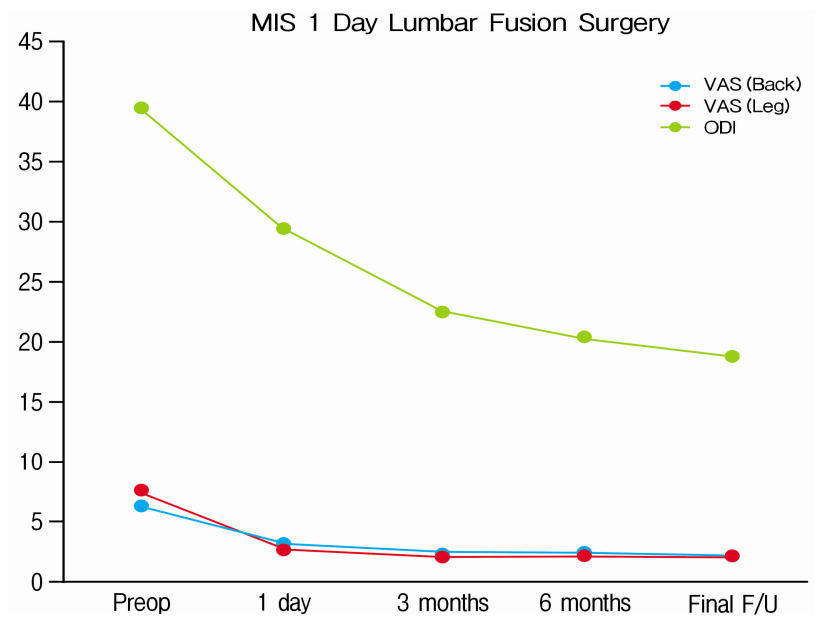

Fig. 13. Patient clinical outcomes.

\section{DISCUSSION}

McGirt et al. ${ }^{8)}$ reported that with 2-level transforaminal lumbar interbody fusion, mean costs associated with perioperative surgical-site infection were significantly lower with MIS than with open procedures. Similar cost-savings have been reported ${ }^{7,14,16,17)}$. The patients' clinical outcomes were assessed via VAS and ODI, but fusion rate was not included in this study. The reason was that the present study sought to examine the viability of 1-day fusion surgery, and fusion rate was intentionally ignored as the objective of this study was to describe the 1-day fusion surgery. However, considering the fact that MIS TLIF was performed for fusion, when compared with conventional TLIF, MIS TLIF appears to achieve similar fusion rates, while reducing blood loss, soft tissue and muscle trauma, postoperative pain, and increasing the speed of recovery ${ }^{9,11,12)}$. It would rather be meaningful to study the fusion rate in the long-term along with other parameters. An epidural catheter was inserted to control postoperative pain. While reducing the pain, it also reduces sensory functions, which may hinder the patient from recognizing postoperative hematoma-induced pain until neurologic deficits, such as motor weakness or cauda equina syndrome, occur. The appropriate dose should be calculated for anesthesia via epidural catheter to ensure that only postoperative pain is reduced.

This study has a limitation regarding patient discharge. In Korea, the government pays $>60 \%$ of all medical costs through the National Health Insurance Service, therefore most patients are discharged after the sutures are removed. The absence of a significant difference in hospital stay between MIS TLIF and the 1-day fusion technique is due to the unique medical system of Korea. To compare cost effectiveness between MIS TLIF and the 1-day fusion technique. Hence, although this study has described a surgical technique that enabled real 1-day discharge, various assessments, such as ambulation, should be emphasized rather than the actual date of discharge ${ }^{2)}$. Early ambulation can lead to early rehabilitation, which in turn leads to early recovery; ultimately, postoperative complications are minimized, leading to effective rehabilitation. National Health Insurance systems differ for each country, so there would be practical differences, but minimally invasive procedures should be studied to develop such surgical techniques.

Many physicians consider Gelform ${ }^{\circledR}$ as a foreign body and worry about mass effect and infection from leaving Gelfoam at the laminectomy site. Although there were no postoperative infections in the present study, the possibility of other issues that may be revealed in a larger number of cases should be noted.

Furthermore, this study did not distinguish patients using anticoagulants, thus did not develop a separate protocol; this should be addressed in the future as well. In addition, preoperative assessment items for 1-day fusion surgery should also be elaborated.

\section{CONCLUSION}

The results indicated good clinical results for the 1-day minimally invasive lumbar interbody fusion surgery without any serious complications. With the development of an effective infection control system for the lumbar interbody fusion surgery, an effective true 1-day lumbar interbody fusion surgery will be possible.

\section{REFERENCES}

1. Choi UY, Park JY, Kim KH, Kuh SU, Chin DK, Cho YE: Unilateral versus bilateral percutaneous pedicle screw fixation in minimally invasive transforaminal lumbar interbody fusion. Neurosurg Focus 35:E11, 2013

2. Choll W. Kim, Todd M. Doerr, Ingrid Y. Luna, Gita Joshua, Sun Ren Shen, Xin Fu, et al: Minimally Invasive Transforaminal Lumbar Interbody Fusion Using Expandable Technology: A Clinical and Radiographic Analysis of 50 Patients. World Neurosurg 90:228-235, 2016

3. Foley KT, Lefkowitz MA: Advances in minimally invasive spine surgery. Clin Neurosurg 49:499-517, 2002

4. Gu G, Zhang H, Fan G, He S, Cai X, Shen X, et al: Comparison of minimally invasive versus open transforaminal lumbar interbody fusion in twolevel degenerative lumbar disease. Int Orthop 38:817-824, 2014

5. Karikari IO, Isaacs RE: Minimally invasive transforaminal lumbar interbody fusion: a review of techniques and outcomes. Spine (Phila Pa 1976) 35:S294-S301, 2010

6. Lee CK, Park JY, Zhang HY: Minimally invasive transforaminal lumbar interbody fusion using a single interbody cage and a tubular retraction system: technical tips, and perioperative, radiologic and clinical outcomes. J Korean Neurosurg Soc 48:219224, 2012

7. Lucio JC, Vanconia RB, Deluzio KJ, Lehmen JA, Rodgers JA, Rodgers W: Economics of less invasive spinal surgery: an analysis of hospital cost differences between open and minimally invasive instrumented spinal fusion procedures during the perioperative period. Risk Manag Healthc Policy 5:65-74, 2012

8. McGirt MJ, Parker SL, Lerner J, Engelhart L, Knight T, Wang MY: Comparative analysis of perioperative surgical site infection 
after minimally invasive versus open posterior/transforaminal lumbar interbody fusion: analysis ofhospital billing and discharge data from 5170 patients. J Neurosurg Spine 14:771-778, 2011

9. Park P, Foley KT: Minimally invasive transforaminal lumbar interbody fusion with reduction of spondylolisthesis: technique and outcomes after a minimum of 2 years' follow-up. Neurosurg Focus 25:E16, 2008

10. Park Y, Ha JW: Comparison of one-level posterior lumbar interbody fusion performed with a minimally invasive approach or a traditional open approach. Spine (Phila Pa 1976) 32:537-543, 2007

11. Peng CW, Yue WM, Poh SY, Yeo W, Tan SB: Clinical and radiological outcomes of minimally invasive versus open transforaminal lumbar interbody fusion. Spine (Phila Pa 1976) 34:13851389, 2009

12. Seng C, Siddiqui MA, Wong KP, Zhang K, Yeo W, Tan SB, et al: Five-year outcomes of minimally invasive versus open transforaminal lumbar interbody fusion: a matched-pair comparison study. Spine (Phila Pa 1976) 38:2049-2055, 2013

13. Shunwu F, Xing Z, Fengdong Z, Xianggian F: Minimally inva- sive transforaminal lumbar interbody fusion for the treatment of degenerative lumbar diseases. Spine (Phila Pa 1976) 35:16151620, 2010

14. Sulaiman WA, Singh M: Minimally invasive versus open transforaminal lumbar interbody fusion for degenerative spondylolisthesis grades 1e2: patient-reported clinical outcomes and costutility analysis. Ochsner J 14:32-37, 2014

15. Walter W. Eckman MD, Lynda Hester PT, Michelle McMillen $\mathrm{RN}$ : Same-day discharge After minimally invasive transforaminal Lumbar Interbody Fusion; A Series of 808 Cases. Clin Orthop Relat Res 472:1806-1812, 2014

16. Wang YW, Lerner J, Lesko J, McGirt MJ. Acute hospital costs after minimally invasive versus open lumbar interbody fusiondata from a US national database with 6106 patients. J Spinal Disord Tech 25:324-328, 2012

17. Won-chul Lee, Jeong-Yoon Park, Kyung Hyun Kim, Sung Uk Kuh: Minimally Invasive Transforaminal Lumbar Interbody Fusion in Multilevel: Comparison with Conventional Transforaminal Interbody Fusion. World Neurosurg 85:236-243, 2016 